\title{
Alter the sheet resistance of carbon nanotube coated cellulose fabric with argon plasma pretreatment
}

\author{
Wei Zhang ${ }^{\text {a, b, * }, ~ S . ~ R a v i ~ P . ~ S i l v a ~}{ }^{\text {b }}$ \\ ${ }^{a}$ School of Materials Science and Engineering, Dalian University of Technology, Dalian 116024, \\ P. R. China \\ ${ }^{b}$ Nanoelectronics Centre, Advanced Technology Institute, University of Surrey, Guildford, Surrey \\ GU2 7XH, United Kingdom
}

\begin{abstract}
Cellulose fabrics were coated with single walled carbon nanotubes (SWCNTs) by a dip-drying process. Scanning electron microscopy (SEM) and Raman spectroscopy analyses indicate the attachment of SWCNTs. The sheet resistance of SWCNT coated fabrics can be altered by modifying the raw cellulose fabrics with low pressure argon plasma. An initial plasma ablation of up to 3 min results in the decrease of sheet resistance, which can be ascribed to the increase of fibre surface roughness, evidenced by SEM analyses. A further increase in the extent of plasma ablation brings about the increase in sheet resistance, which is associated with the incorporation of oxygen functionalities, supported by the data obtained on colour strength measurements.
\end{abstract}

Key words: cellulose fabric, carbon nanotube, plasma, electrical conductance

\section{Introduction}

Monitoring the concentration of chemicals, gases and vapours is of great importance for environmental protection, control of chemical processes, space exploration, as well as

\footnotetext{
* Corresponding author. E-mail: zhangweileeds@ hotmail.com, Tel: +44 1483 686102, Fax: +44 1483689404.
} 
agricultural and medical applications [1-3]. Commonly used gas sensing materials mainly include metal oxide semiconductors, such as tin oxide, zinc oxide, titanium oxide, aluminum oxide [4], and conductive polymers and organic semi-conductors, for instance, polyaniline, polypyrrole and poly (3,4-ethylenedioxythiophene) [5]. Difficulties encountered with the former are lack of flexibility, poor response speed, and operated at elevated temperatures; while the latter can only provide limited sensitivity [6-8]. Clearly, there is a need to develop novel sensors that allow for operation at room temperatures with high sensitivity and short response time.

Since the most common gas sensing principle is the adsorption and desorption of gas analytes on sensing materials, it is fairly understandable that the sensitivity will be improved if the contact interfaces between gas analytes and sensing materials are raised. As a consequence, carbon nanotubes (CNTs) have been extensively exploited as promising candidates for fabricating gas and chemical sensors due to their extremely high surface-to-volume ratio and hollow geometry $[9,10]$. The sensing mechanism of CNTs to gases relies on the fact that when adsorbed on CNTs the analyte gas molecules can act as dopants, shifting the Fermi energy of CNTs and changing the band-structure of CNTs. Through the operation of electron transfer, the electrical conductance of CNTs will either increase or decrease, functioning as both sensing materials and transducers [11]. Indeed, CNT based sensors that can be operated at room temperature with high sensitivity have already been demonstrated. These sensors have displayed good performance on the detection of nitrogen dioxide, ammonia, alcohol, dimethyl methylphosphonate, thionyl chloride, ozone, nitrotoluene etc [1].

However, most, if not all, of these sensors are manufactured on rigid substrates, which exclude themselves from the consideration of portable applications. To improve the flexibility and wearability, cellulose fabrics have been successfully used as substrates to make sensors [12]. And it was found that the sensitivity of CNT-coated cellulose chemiresistor to ammonia gas is highly dependent on the initial resistance of the sensor [13]. The initial resistance of sensor may 
also determine the accuracy requirement for power control and affect the signal-to-noise ratio of sensors. Undoubtedly, a technique that can be adopted to tune the initial resistance of CNT coated cellulose fabrics is highly desired. As documented, plasma modification can impose effects on the surface morphology and chemical composition of fabrics [14-16], which may affect the interactions between CNTs and fabrics in the coating process, and, in turn, the resistance of CNT coated fabrics. With all these in mind, in this study, we attempt to alter the resistance of CNT coated cellulose fabrics by modifying the raw cellulose fabrics with argon plasma.

\section{Experimental}

\subsection{Plasma modification}

Cellulose fabrics (Whaleys, UK) were first scoured at $60^{\circ} \mathrm{C}$ for $15 \mathrm{~min}$ in $2 \mathrm{~g} / \mathrm{cm}^{3} \mathrm{Na}_{2} \mathrm{CO}_{3}$ and $1 \mathrm{~g} / \mathrm{cm}^{3} \mathrm{MFB}$ (non-ionic detergent, BASF, Germany) with a liquid ratio of 1:20. After drying in air, the fabrics were treated with an argon plasma (gas flow rate: $20 \mathrm{sccm}$ ) on a RF plasma barrel reactor (Emitech K1050X Plasma Asher, Quorum Technologies, UK) at a vacuum of 50 $\mathrm{Pa}$ and a $100 \mathrm{~W}$ discharge power, and with an exposure time of $0.5,3,5$, and $10 \mathrm{~min}$.

\subsection{Attachment of CNTs}

The aqueous mixture of 2 wt. \% dodecylbenzenesulfonic acid, sodium salt (Sigma-Aldrich, UK) and $0.1 \times 10^{-3} \mathrm{~g} \mathrm{~cm}^{-3}$ single walled carbon nanotubes (SWCNTs, NanoLab, US) were sonicated in a Cole-Parmer Ultrasonic processor (UK) for 20 min with a power of $120 \mathrm{~W}$. Subsequently, the plasma treated cellulose fabrics were immersed and kept in the SWCNT suspension for $1 \mathrm{~h}$. The resulting fabric was then taken out of the suspension, air-dried, and the whole procedure was repeated for 4 cycles.

Fig. 1 demonstrates the results of fastness investigation. The SWCNT coated fabrics were immersed in either water (Fig. 1a) or ethanol (Fig. 1b) and heated to $40^{\circ} \mathrm{C}$ for $15 \mathrm{~min}$; meanwhile, 
the fabrics were rolled and squeezed several times. The SWCNT coated fabrics were also adhered with a commercial transparent tape (Fig. 1c).

\subsection{Characterization}

Scanning electron microscope (SEM) images were obtained on a FEI Quanta 200 (FEI). To alleviate the charging phenomena in SEM observations, stemming from the insulating nature of fabrics, the raw cellulose fabric and the plasma modified cellulose fabrics were coated with gold in a JLS Sputtering System (JLS Designs) for 2.5 min with the following operating parameters: Power: $1000 \mathrm{~W}$, Voltage: $300 \mathrm{~V}$, Current: 60 A. All the SWCNT coated fabrics were observed as they were. Raman spectra were obtained on a Renishaw System 2000 microRaman spectrometer (Renishaw) with an excitation at $782 \mathrm{~nm}$. The sheet resistance of SWCNT coated cellulose fabrics was measured on a four point probe stand $(K \& S)$.

\subsection{Colour strength measurement}

$C I E L^{*} a^{*} b^{*}$ and the reflectance $\left(R_{\lambda}\right)$ of the raw, plasma modified and SWCNT coated cellulose fabrics were analyzed with a Datacolor SF600 Spectraflash colour spectrophotometer connected to a PC using DCI Colour Tools software from 400-700 nm with an increment of 20 $\mathrm{nm}$ under a $\mathrm{D} 65 / 10^{\circ}$ illuminant. From the reflectance at a given wavelength, the colour strength $(K / S)$ was calculated using the Kubelka-Munk equation \{Eq. (1)\} [17].

$$
K / S=\frac{\left(1-R_{\lambda}\right)^{2}}{2 R_{\lambda}}
$$

The colour strength function $\left(f_{k}\right)$ from 400 to $500 \mathrm{~nm}$ was calculated using Eq. (2), where $\overline{\mathrm{x}}_{10, \lambda}, \overline{\mathrm{y}}_{10, \lambda}$ and $\overline{\mathrm{z}}_{10, \lambda}$ are the colour matching functions for the $10^{\circ}$ standard observer at each wavelength measured.

$$
\mathrm{f}_{\mathrm{k}}=\sum_{\lambda=400}^{\lambda=500}(\mathrm{~K} / \mathrm{S})_{\lambda}\left[\overline{\mathrm{x}}_{10, \lambda}+\overline{\mathrm{y}}_{10, \lambda}+\overline{\mathrm{z}}_{10, \lambda}\right]
$$




\section{Results and discussion}

\subsection{Attachment of SWCNTs onto cellulose fabrics}

SEM images of the raw cellulose fibres, plasma modified cellulose fibres and SWCNT coated cellulose fibres are presented in Fig. 2. For plasma modified cellulose fibres only 0.5 min and 10 min plasma treated samples are shown. In comparison with the raw cellulose fibres, an initial exposure to argon plasma does not induce a significant change in the surface features of fibres (Fig. 2b). A further increase in plasma exposure time causes more severe damage of fibre surfaces, and an etched pattern with hills and valleys becomes dominated in fibre surface profile (Fig. 2c). After the coating with SWCNTs, the up-and-down surface feature of cellulose fibres becomes indistinguishable, and instead a relatively smooth surface is observed (Fig. 2d). It is also noticeable that this smooth surface is completely different from that of the raw cellulose fibres, wherein fibrillar structures prevail. A combination of these results indicates the occurrence of coating.

To confirm the presence of SWCNTs on fibre surfaces, Raman spectroscopies were carried out and the Raman profiles of SWCNT coated fabrics are depicted in Fig. 3. The characteristic bands of SWCNTs, namely, radical breathing mode (RBM, 160-200 $\left.\mathrm{cm}^{-1}\right), \mathrm{D}\left(\sim 1310 \mathrm{~cm}^{-1}\right), \mathrm{G}$ $\left(\sim 1590 \mathrm{~cm}^{-1}\right)$, and $\mathrm{G}^{\prime}\left(\sim 2600 \mathrm{~cm}^{-1}\right)$, are all clearly identifiable, suggesting the incorporation of SWCNTs onto fibre surfaces [18].

To investigate the fastness of the SWCNT coatings, the SWCNT coated fabrics were rolled up and squeezed in water and ethanol in conjunction with heat treatment, no clear SWCNT precipitating was observed (Fig. 1a and b). The SWCNT functionalized fabrics were also adhered with a commercial transparent tape; no obvious peeling off of SWCNTs from fabric was visible on the transparent tape (Fig. 1c). These results indicate the firm attachment of SWCNTs onto fabrics.

\subsection{Colour strength $(K / S)$ of plasma modified cellulose fabrics}


In experiments, it was observed that all the plasma modified fabrics become somewhat yellowish. To characterize this phenomenon, the colour strength of the raw cellulose fabric and fabrics modified with argon plasma for $0.5,3,5,10 \mathrm{~min}$ is calculated and plotted in Fig. 4. In contrast to the raw cellulose fabric, all the plasma modified fabrics show higher colour strength in the range of $400-500 \mathrm{~nm}$, and the colour strength increases with the prolonged plasma ablation. This means all plasma modified fabrics exhibit enhanced absorption of the bluish light, and this enhancement is strengthened with the increasing extent of plasma ablation. The absorption of blue light can render the fabrics a yellow colour. To give a better correlation between the measured and visual assessment, the colour strength function $\left(f_{k}\right)$ in the range of $400-500 \mathrm{~nm}$ is plotted in the inset of Fig. 4. When the plasma modification duration is increased from 0 min (the raw cellulose fabric) to $10 \mathrm{~min}$, the colour strength function increases from 0.21 to 1.00 monotonously. This result strongly indicates that the prolonged plasma modification can incur a deeper yellow hue in fabrics.

The CIE $L^{*} a^{*} b^{*}$ of the fabrics were also recorded and are listed in Tab. 1, which shows that $b^{*}$ is 0.98 for the raw cellulose fabric and increases to 11.61 when the plasma ablation duration is increased to $10 \mathrm{~min}$. In $C I E L^{*} a^{*} b^{*}$ colour space, the coordinate $b^{*}$ suggests the position between yellow and blue. Negative values indicate blue and positive values indicate yellow; and the larger the $b^{*}$ value, the more yellowish the substance. This result is in good agreement with the analysis with regard to the colour strength and the colour strength function.

The above discussion can explain why all the plasma modified cellulose fabrics look yellowish to human colour response. The yellowing of celluloses may be attributed to the oxidization of celluloses incurred by the plasma modification, yielding the presence of aldehyde groups, most likely, in the form of furan derivatives [19]. It is also of interest to note that both the colour strength and $b^{*}$ increase with the prolonged plasma ablation; this is not surprising since the prolonged treatment may anchor more aldehyde groups to the fabrics. 


\subsection{Altering the sheet resistance by plasma modification}

The sheet resistance of SWCNT coated cellulose fabrics with up to 4 times of immersion was measured and is plotted in Fig. 5 as a function of the plasma exposure duration. Fig. 5 exhibits that all the plasma modified cellulose fabrics exhibit lower sheet resistance values than the raw fabrics after the same time of immersion in the SWCNT dispersion, which may be explained in terms of the increase of fibre surface roughness. As SEM images display (see Section 3.1), hills and valleys are generated on fibre surfaces by plasma modification, facilitating the improvement of contact interfaces between SWCNTs and cellulose fabrics and thereby the attachment of SWCNTs onto fabrics.

It is also apparent that for the fabric modified for a given duration the sheet resistance drops with the increase in the number of immersion cycle. This is not out of expectation since a larger quantity of SWCNTs will become attached to cellulose fabrics by hydrogen bonding and van deer Waals forces with the repeated immersion. As a consequence, the electrically conductive networks in cellulose fabrics developed by SWCNTs become proliferated. It is worth mentioning that the electrically conductive networks form not only by the direct contact of metallic or semiconducting SWCNTs but also electron jumping between them. When two conducting particles are separated by a distance in the order of nanoscale, the energy barrier for the electron jumping diminishes substantially and the electrons can easily jump from one particle to another. From the viewpoint of electrical conduction, this is equal to the direct contact of particles [20].

For the cellulose fabrics that undergo the same number of immersion, the sheet resistance is also associated with the plasma ablation duration. An initial exposure of cellulose fabrics to the plasma ablation results in the decrease of the sheet resistance, and the sheet resistance reaches its minimum after 3 min modification; whereas a further increase in the plasma modification gives rise to the increase in the sheet resistance. As the sheet resistance is determined by the conductive networks developed by the adsorbed SWCNTs, this variation trend should be 
intimately related to the amounts of SWCNTs that are adsorbed on fabrics. Since colour strength $(\mathrm{K} / \mathrm{S})$ is a function of the reflectance of fabrics and reflects the amounts of SWCNTs presented, K/S of SWCNT coated fabrics after 4 immersions were measured and is presented in Fig. 6 . The $\mathrm{K} / \mathrm{S}$ increases with the increase of plasma ablation time from $0 \mathrm{~min}$ to $3 \mathrm{~min}$, and then drops when the plasma ablation time is increased to $10 \mathrm{~min}$. This is fairly consistent with the observed variation of sheet resistance discussed above.

The observed variation in sheet resistance with plasma exposure time may be ascribed to the incorporation of oxygen functionalities, quite possibly in the form of aldehyde groups, as discussed in Section 3.2. It is worthy of mentioning that no oxygen gas was introduced on purpose in argon plasma modification. However, it is hard to evacuate all the air out of the plasma chamber and create an entirely air-free environment, and the present oxygen, even at a fairly small amount, still represents a very surface reactive species, which can react with the radicals produced by argon plasma and be implanted onto fibre surfaces [21,22]. The implanted oxygen functionalities impair the attachment of SWCNTs in that these aldehyde groups are hydrophilic in nature, while SWCNTs are hydrophobic, creating a higher energy barrier for SWCNTs from getting close and attached to fibre surfaces. With the increase in the extent of plasma ablation, more hills and valleys will be created on fibre surface; in the meanwhile more oxygen functionalities will be incorporated. This means the effect of plasma modification on the attachment of SWCNTs is actually the competing result of these two effects. With an initial exposure to argon plasma, the increase in surface roughness prevails and more SWCNTs become attached in the subsequent dip procedure; whereas a further increase in the extent of plasma ablation gives rise to the abundance of hydrophilic oxygen groups on fibre surfaces, impeding the attachment of SWCNTs. Consequently, a flex point phenomenon was observed in the diagram of the variation of sheet resistance versus plasma ablation time. 


\section{Conclusions}

It was demonstrated that the sheet resistance of SWCNT coated cellulose fabrics can be altered by the modification of the raw cellulose fabrics with argon plasma. This is ascribed to the effect of plasma modification on the morphology and chemical composition of fibre surfaces. An initial exposure to plasma generates hills and valleys on fibre surface, facilitating the attachment of SWCNTs. A further increase in plasma ablation results in the abundance of oxygen functionalities on fibre surface, hampering the attachment of SWCNTs. The overall sheet resistance of SWCNT coated cellulose fabrics is the competing outcome of these two effects.

\section{Acknowledgements}

This work was supported by National Natural Science Foundation of China (Granted No. 51105051). We would like to thank Dr. Parikshit Goswami (University of Leeds), Mr. Yee Yuan Tan, and Mr. K. D. G. I. Jayawardena (University of Surrey) for their useful discussions.

\section{References}

[1] T. Zhang, S. Mubeen, N. V. Myung, M. A. Deeshusses, Recent progress in carbon nanotubebased gas sensors, Nanotechnology 19 (2008) 332001.

[2] Y. Wang, J. T. W. Yeow, A review of carbon nanotubes-based sensors, Journal of Sensors 9 (2009) 2289-2319.

[3] F. P Rouxinol, R. V. Gelamo, S. A. Moshkalev, Carbon Nanotubes, Edited by: Jose Mauricio Marulanda, Gas sensors based on decorated carbon nanotubes, 357-374, InTech, 2010.

[4] S. K. Koh, H. J. Jung, S. K. Song, W. K. Choi, D. Choi, J. S. Jeon, Sensor having tin oxide thin film for detecting methane gas and propane gas, and process for manufacturing thereof, US patent 6,059,937 (2000).

[5] H. Bai, G. Shi, Gas Sensors Based on Conducting Polymers, Sensors 7 (2007) 267-307.

[6] J. Li, Y. Lu, Q. Ye, M. Cinke, J. Han, M. Meyyappan, Carbon nanotube sensors for gas and organic diction, Nano Lett. 3 (2003) 929-933.

[7] M. Y. Faizah, Room temperature multi gas diction using carbon nanotubes, Eur. J. Sci. Res. 35 (2009) 142-149.

[8] S. Peng, J. O'Keeffe, C. Wei, K. Cho, Carbon nanotube chemical and mechanical sensors, Conference paper for the $3^{\text {rd }}$ International Workshop on Structural Health Monitoring, Stanford University, Palo Alto, California, September 12-14, 2001, 1-8.

[9] A. Modi, N. Koratkar, E. Lass, B. Wei, P. M. Ajayan, Miniaturized gas ionization sensors using carbon nanotubes, Nature 424 (2003) 171-174. 
[10] I. Sayago, E. Terrado, E. Lafuente, M. C. Horrillo, W. K. Maser, A. M. Benito, R. Navarro, E. P. Urriolabeitia, M. T. Martinez, J. Gutierrez, Hydrogen sensors based on carbon nanotubes thin films, Synthetic Met. 148 (2005) 15-19.

[11] J. Li, Y. Lu, Q. Ye, L. Delzeit, M. Meyyappan, A gas sensor array using carbon nanotubes and microfabrication technology, Electrochem. Solid-State Lett. 8 (2005) H100-H102.

[12] B. S. Shim, W. Chen, C. Doty, C. Xu, N. A. Kotov, Smart electronic yarns and wearable fabrics for human biomonitoring made by carbon nanotube coating with polyelectrolytes, Nano Lett. 8 (2008) 4151-4157.

[13] W. Zhang, Y. Y. Tan, C. W. Wu, S. R. P. Silva, Self-assembly of single walled carbon nanotubes onto cotton yarn: Towards the production of conductive yarn and ammonia sensor, Particuology, 2012, doi:10.1016/j.partic.2011.06.011.

[14] A. Riza, Y. Abbas, Low temperature dyeing of plasma treated luxury fibres. Part I: Results for Mohair (Angora Goat), Fibres Text. East. Eur. 19 (2011) 84-89.

[15] N. De. Geyter, R. Morent, C. Leys, Surface modification of a polyster non-woven with a dielectric barrier discharge in air at medium pressure, Surf. Coat. Technol. 201 (2006) 24602466.

[16] C. Cheng, L. Y. Zhang, R. J. Zhan, Surface modification of polymer fibre by the new atmospheric pressure cold plasma jet, Surf. Coat. Technol. 200 (2006) 6659-6665.

[17] R. McDonald, Industrial pass/fail colour matching. Part III. Development of a pass/fail formula for use with instrumental measurement of colour-difference. J. Soc. Dyers Color. 96 (1980) 486-497.

[18] M. S. Dresselhaus, G. Dresselhaus, R. Saito, A. Joriod, Raman spectroscopy of carbon nanotubes, Physics Reports 409 (2005) 47-49.

[19] M. Albeck, A. Ben-Bassat, M. Lewin, Part II: The influence of functional groups and the nature of the yellowing, Text. Res J. 35 (1965) 935-942

[20] W. Zhang, A. Dehghani-Sanij, R. S. Blackburn, Carbon based conductive polymer composites, J. Mater. Sci. 42 (2007) 3408-3418.

[21] R. Dorai, M. J. Kushner, A model for plasma modification of polypropylene using atomospheric pressure discharges, J. Phys. D Appl. Phys. 36 (2003) 666-685

[22] Natural Fibres Synthetic Fibres-Cotton. http://www.swicofil.com/products/001cotton.html. Accessed 1 Sept 2011. 


\section{Figure captions:}
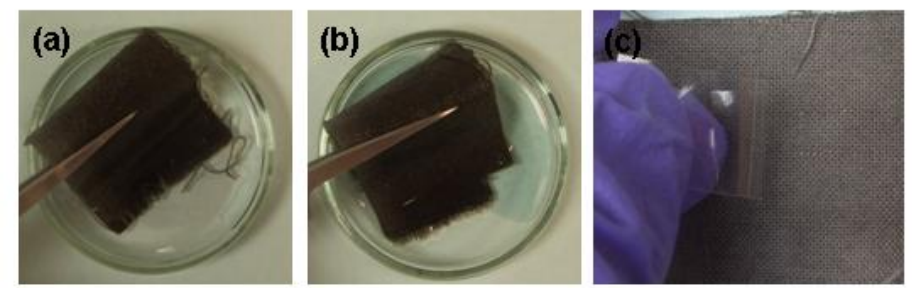

Fig. 1 SWCNT coated cellulose fabrics washed in water (a) and ethanol (b). The SWCNT coated cellulose fabric after adhesion with a transparent tape (c)

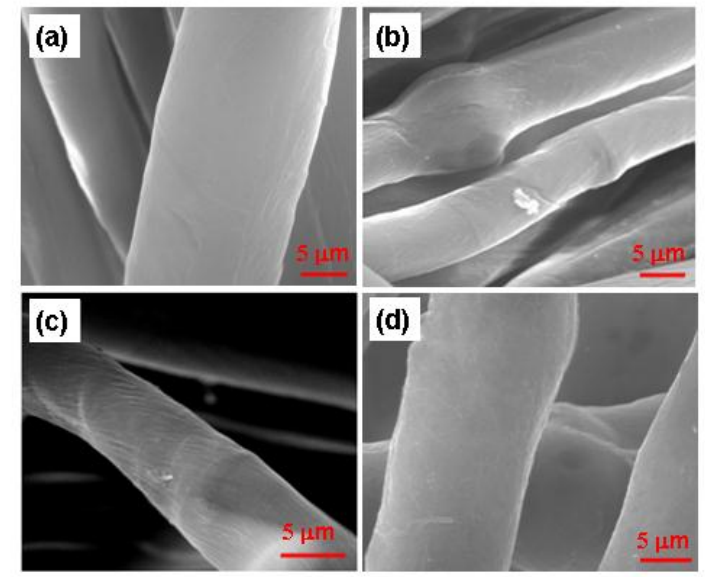

Fig. 2 SEM images of raw cellulose fibres (a), argon plasma modified cellulose fibres (b) 0.5 min, (c) 10 min, and SWCNT coated cellulose fibres after 4 times of immersion (d)

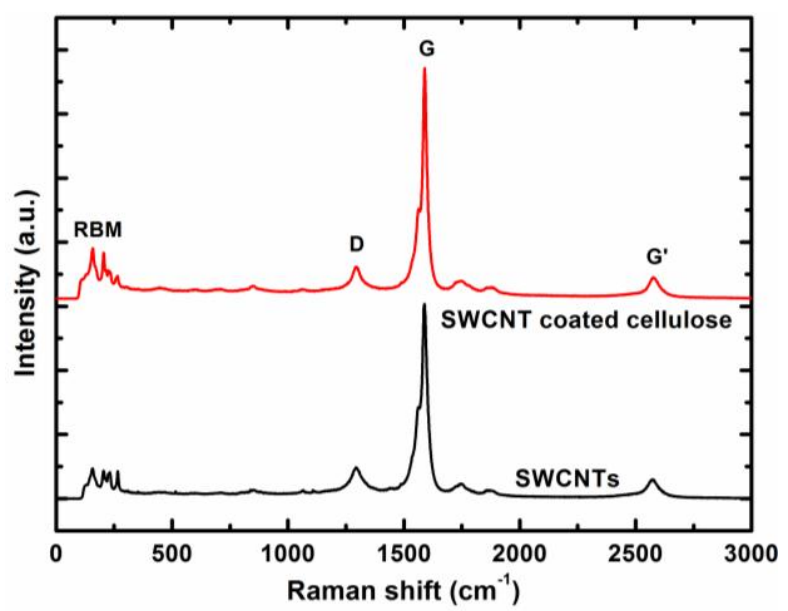

Fig.3 Raman spectra of SWCNTs and SWCNT coated cellulose fabrics 


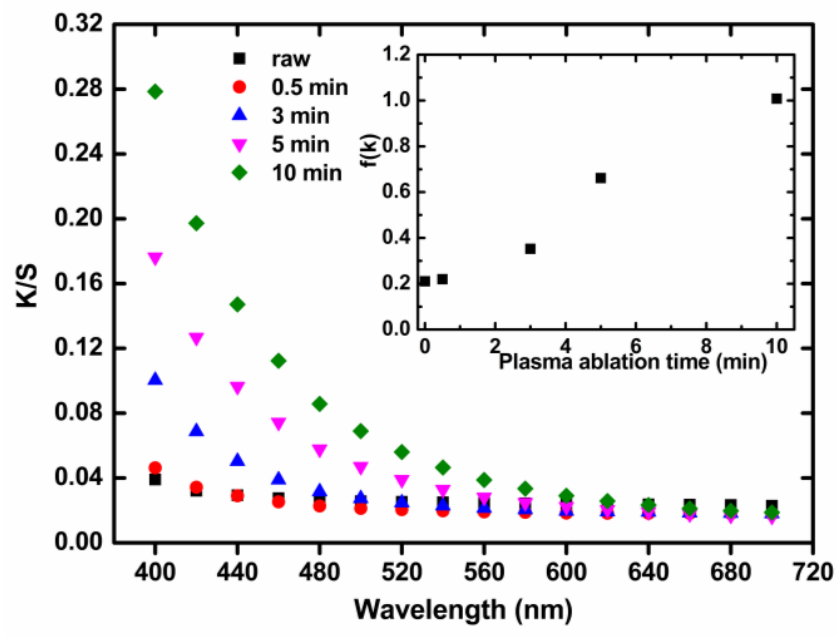

Fig. 4 The colour strength over full visible spectrum of raw and argon plasma modified cellulose fabrics. Insets: the colour strength function in the range of $400-500 \mathrm{~nm}$ of raw and argon plasma modified cellulose fabrics against plasma ablation time

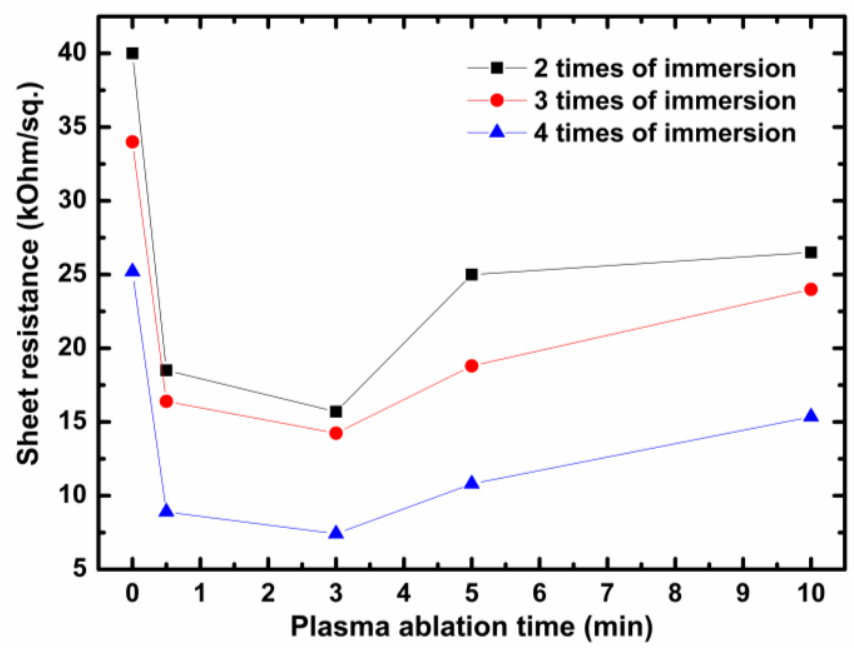

Fig. 5 Sheet resistance of SWCNT coated cellulose fabrics with up to 4 times of immersion against plasma ablation time 


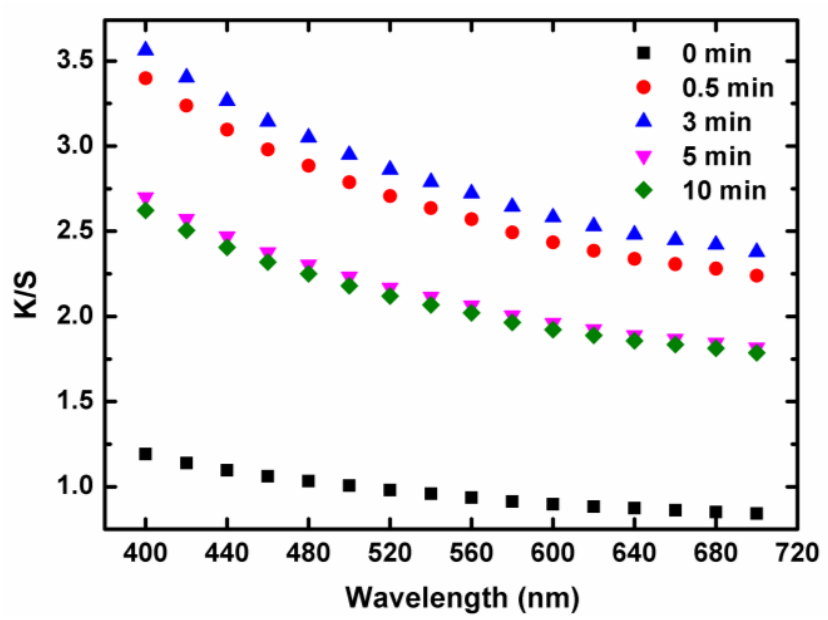

Fig. 6 The colour strength over full visible spectrum of SWCNT coated cellulose fabrics after 4 times of immersion 
Tab. $1 C I E L^{*}, a^{*}, b^{*}$ of raw cellulose fabric and fabrics modified with argon plasma for different duration

\begin{tabular}{cccccc}
\hline & $0 \min$ & $0.5 \min$ & $3 \min$ & $5 \min$ & $10 \min$ \\
\hline$L^{*}$ & 91.70 & 92.56 & 91.97 & 90.58 & 88.99 \\
$a^{*}$ & -0.12 & -0.38 & -0.83 & -0.05 & 0.32 \\
$b^{*}$ & 0.98 & 2.11 & 5.05 & 9.09 & 11.61 \\
\hline
\end{tabular}

